International Journal of Engineering \& Technology, $7(3.29)(2018)$ 95-102
International Journal of Engineering \& Technology
Website: $w w w . s c i e n c e p u b c o . c o m / i n d e x . p h p / I J E T$
Research paper

\title{
Enabling Business transformation through enterprise architecture and the knowing cycle
}

\author{
Hanlie Smuts ${ }^{1}$, Busisiwe Juleka ${ }^{1}$ \\ ${ }^{1}$ University of Pretoria \\ *Corresponding author E-mail: hanlie.smuts@up.ac.za
}

\begin{abstract}
Business transformation in organizations is imperative due to the rapid and complex nature of the changes in business environments. Organizations are required to consider new strategies, innovative products and services, and adapt to disruptive and different ways of doing business to remain competitive. The nature of these changes requires holistic and strategic approaches, such as enterprise architecture, to be efficiently managed. Furthermore, the knowledge that exists in the organization, in any way that it is available, is required to develop an enterprise architecture. Knowledge management, and in particular the knowing cycle, guides the sense making capacity of an organization to understand the change that is taking place, the creation of new knowledge to address the change and informs decision making. Therefore, the purpose of this paper is to consider how enterprise architecture in association with the knowing cycle enables business transformation. Based on the findings of this study, we present a business transformation framework grounded in the alignment of the sense making processes to the establishment of an architecture vision to bracket the change situation. By applying such a framework, organizations may be guided to the best possible initiatives in order to enable business transformation and articulate the development of a future state architecture.
\end{abstract}

Keywords: Business Transformation; Enterprise Architecture; Knowing Cycle; Knowledge.

\section{Introduction}

The complexity of business in the information age, where information is easily available especially with the ubiquitous presence of information technology yet the meaning of information is often ambiguous, forces organizations to change [1]. Irrespective of whether these organizational changes are long-term or short-term and consist of multiple, interrelated components, they have a structural aspect that requires strategic management by using the information that is present in the organization enabling its capacity to grow and adapt [2-3]. The strategic management components include the definition of organizational strategies, goals, business products, business services, business processes, and ultimately their business standard operating procedures, all forming part of their enterprise model [4]. An enterprise model "represents the resources found in the enterprise and its environment, together with the processes in which they participate" [5-2] and is utilized to reason and communicate about the organization as part of an enterprise architecture (EA) project [5]. One of the business imperatives of such an EA project is the facilitation of business transformation $[1,6]$. The purpose of EA in this context is to optimize the often fragmented legacy of processes (both manual and automated) across the organization into an integrated environment that is responsive to change and supportive of the delivery of the business strategy [7]. It is also a form of knowledge creation for an organization [8].

One of the key dependencies for an organization to survive the complexities of change is to be constantly aware of the changes in its external environment. Consequently, organizations need to understand what it knows about itself, organizations need to appreciate the insights into their environment, interpret these, and respond ap- propriately [3]. This ensures that all organizational stakeholders, including organizational management, understand their organization and what affects it [9]. In order to preserve their legitimacy within the business environment, organizational management must take rational and informed decisions regarding the organizational change [3]. Furthermore, making sense of existing knowledge and the creation of new knowledge is vital in influencing the decisions taken by organizational management in order to keep the organization relevant [3]. Such new knowledge created within an organization, enables innovation which encourages the development of new capabilities, enhances existing products and services, and improves business processes [10].

However, little research has been done to show the role of knowledge management concepts and the knowing cycle in the development of enterprise architecture [3]. Therefore, the objective of this paper is to analyze and describe how enterprise architecture in association with the knowing cycle enables business transformation. The findings of this study are collated into a business transformation framework. Section II of the paper provides the background, section III describes the method followed in conducting the research, section IV reflects on the research findings and the proposed framework and section $\mathrm{V}$ concludes the paper.

\section{Background}

Changes in the external environment of organizations powers rapid and highly complex changes in organizational activities, products and services with the aim to satisfy growing customer needs, elevate organizational structures, and optimize information technology infrastructure [1], [6], [11]. The nature of these changes is transformational and their impact such that they alter the strategic direction 
of an organization, that they bring about new opportunities [12], and that they innovate new products [12-13]. They call for the use of organizational knowledge to make the right management decisions [13]. The management of such an all-inclusive change, poses a challenge to the organization and requires the application of a holistic approach, such as EA $[6,12]$. As the EA baseline and target architecture illustrate how the business operates and how it will operate in future, it forms an integral part of the business transformation plan [14].

In the next sections we will explore business transformation and EA further, as well as providing and overview of knowledge management $(\mathrm{KM})$ and in particular, the knowing cycle, in the context of business transformation and EA.

a) Business Transformation and Enterprise Architecture

Business transformation refers to "a fundamental change in organizational logic which resulted in or was caused by a fundamental shift in behaviours" [15]. Business transformation is characterized by external change drivers, a change in why organizations do business and how the business is done, and a change in leadership and culture [9].

Prahalad \& Oosterveld [16] present 5 characteristics that define a successful business transformation. Firstly, it is driven by new opportunities which lead to new strategies and management processes. It is not just about cost reduction, increase in revenue, or reengineering. Secondly, it involves the whole organization implying that the new opportunity will come with new perceptions that must be shared by the entire organization. Thirdly, the tacit organizational values and beliefs that organizational members may have, must be managed as these tacit organizational values influence enactment. Fourthly, it requires that organizational members build new skills and knowledge to be able to participate in new markets and sustain a competitive advantage. Lastly, it must be cemented with new management processes such as performance evaluation, talent management, rewards and recognitions, and product development.

Successful business transformation is underpinned by a transformation plan and in the literature multiple approaches for business transformation plans are offered. Stoop, et al., [2] detail the development of a business transformation plan as consisting of three phases, namely: (1) initiation phase, (2) development phase, and (3) delivery phase. The initiation phase ensures a focused approach that attempts to assess the organizational situation, including evaluation of available resources, analyzing the stakeholders, and measuring the capacity for change. This phase clarifies the underlying motivations and the goals for the plan. The development phase delivers the business transformation plan to the stakeholders. It is essential that the right stakeholders are involved in this phase to contribute organization-specific knowledge to assist in crafting the target situation. To conclude this phase, the executive must take ownership of, and approve the plan. The delivery phase is concerned with making sure that the business transformation plan as drafted in the development phase is implemented. The business transformation plan can therefore be referred to as a master plan for all change activities in an organization. Saini \& Khurana [17] present 4 stages of a transformation plan, namely (1) reframe, (2) restructure, (3) revitalize and (4) renew. Reframe refers to the creation of a new future vision, the mobilization of the organization in support of this vision, as well as the development of a measurement system to track performance in this regard. The redesign of the value chain and organization in accordance with the revised business strategy and in alignment with its new business model, form part of the restructure stage. The revitalize stage is applied in order to kindle growth through value proposition revitalization, the invention of new business and the revisiting of working rules. The final stage, renew, points to the advancement of the people components in the organization through the update of the reward systems, by encouraging individual learning and through the development of the organization. According to Stiles et al [18], every business transformation is an iterative process consisting of 4 phases in recurring cycles: (1) envision, (2) engage, (3) transform and (4) optimize. The envision phase creates the case for change and oversees the construction of the revised vision and strategy. During the engage phase people are empowered to act on the vision and to plan the related efforts. The transform phase changes relevant planned elements such as the processes, technology, behaviour, culture, values etc. Stability is created with the optimize phase where the transformation is internalized, institutionalized and optimized. Irrespective of the business transformation plan steps followed, the transformation process should be continuous; no organization can survive over the long-term if it does not re-invent itself [17]

The success of a business transformation initiative is dependent on how change enablers are integrated to the organization's enacted strategy and how well the steps of the transformation plan are executed [15]. Stoop, et al. [2] outline specific steps regarding how the business transformation plan may be delivered. The first step is to elaborate the strategy and objectives which forms the foundation for a business transformation portfolio as it details the mission, vision and specific objectives for the organization. The strategy provides direction on the targeted transformation. The next step is to formulate the guiding principles. In order to be able to assess the current organizational landscape, guiding principles are required as they would provide enough clarity on the expectations of the target landscape. These principles are informed by the strategy and take the form of policy documents. Following the formulation of the guiding principles, both the current and target situation must be outlined. At this point the guiding principles are used to guide the analysis of the current situation, gaps are identified which direct the outlining of the target situation. This is done through the description and development of an enterprise architecture model. The enterprise architecture model provides control of the change. The next step is to formulate the action items. Action items are derived from the gap analysis i.e. the difference between the current and target situation, as well as from the analysis of the current situation where constraints and opportunities for improvement are identified. The last step is to draft the business transformation portfolio. The collection of the action items described in the previous step are grouped into projects and prioritized into a roadmap focused on delivering the business transformation.

The enacted strategy is a competitive strategy which takes into account organizational external and internal forces that interplay to transform the performance of an organization [15]. In the next section, we provide an overview of the organization and EA.

b) Enterprise Architecture and the Organization

Enterprise Architecture (EA) is the manner in which an organization is structured, reflecting the relationship between business processes and information technology infrastructure for delivering value to customers [19]. EA "serves as the blueprint for the system and the project that develops it" [20:18]. More specifically, EA is holistic in scope, enterprise-wide in its coverage and bridges the gap between strategic planning and execution [19, 21, 22]. The term 'enterprise' is the highest level of description of an organization which covers all of its functions and goals, inclusive of a structure of components, their inter-relationships, principles and guidelines governing their design and evolution over time [7]. Another description of EA is a process that "results in the creation and iterative refinement of multiple artefacts that collectively define a future EA which will identify the gaps between the current state and future state" [23] [3].

EA it is able to identify, scope and scale the required change in organizational elements as it addresses all organizational activities and the supporting information technologies [21]. Hence, EA artefacts are in the form of models, catalogues, matrices and documentation held in a managed architecture repository, which acts as knowledge management tool [24]. Furthermore, EA artefacts are not meant to be static, as this will inhibit the competitiveness of the knowledge that is created [23].

Zachman [25] in his seminal publication 'The framework for information systems architecture' recognizes the importance of architecture for the success of an organization. Organizations that have developed a good EA practice can easily identify required changes, estimate the impact of change and understand it [26]. A good architecture facilitates stable and flexible innovation and change, whilst also balancing business requirements and translating organizational 
strategy to day-to-day operations [11]. Moreover, it is a valuable asset that captures the interactions and interconnections of a business with its external environment which includes customers and suppliers, as well as its computing environment $[11,21]$. EA provides a path to bring into line the IS environment with the business reality and the strategic goals, or to assess the alignment for greater competitive advantage [27].

EA is a mechanism of creating a knowledge base through fulfilling the requirements of an enterprise architecture framework for an organization and this knowledge base requires management [25]. Such management may be facilitated by selecting a knowledge management tool based on organizational requirements with the aim to not only manage EA knowledge, but also to facilitate the updating and reporting for decision making $[28,29]$. In the next section we consider knowledge and the management of knowledge in organizations.

c) Knowledge and its Management in the Organization The concept of knowledge has long been investigated by epistemologists and as such many definitions of knowledge are reflected in the literature [30]. De Long [10] mentions two categories of knowledge that managers in organizations ought to keep in mind: (1) location of knowledge i.e. knowledge at individual, group, or organizational levels; and (2) the type of knowledge characterized by either tacit or explicit knowledge. Choo [31] further expands on the types of knowledge distinguishable by structure:

- Explicit knowledge is articulated formally and is easily communicated;

- Tacit knowledge cannot be articulated;

- Implicit knowledge can be articulated, but has not been articulated;

- Cultural knowledge is formed by beliefs based on observation, experience;

- Strategic knowledge is knowing when to do something and why to do it.

Knowledge is a part of a number of important assets an organization may claim and must therefore also be managed [32]. One of the ways to manage knowledge is to see it as a business service and to ensure the systematic acquisition, synthesis, and sharing of insights and experiences [33].

Knowledge management $(\mathrm{KM})$ is a multifaceted concept based on the nature and types of knowledge, although there are various and varied definitions for the notion [34]. Sveiby [35: 37] defines the management of knowledge as "the art of creating value by leveraging intangible assets". Meyer and Botha [36:278] define it as "the management of corporate processes designed to create, disseminate and protect knowledge in support of sound decisions leading to profit". Godbout [37] defines knowledge management by suggesting that it is not knowledge that gives the competitive edge, but rather the capacity to transform knowledge into competencies and replicate know-how. Agresti [38:3] defines knowledge management as "the practice of transforming the intellectual assets of an organization into business value". However, for the purpose of this paper, the following definition of knowledge management, as suggested by Choo [39], will be used: "[A] framework for designing an organization's goals, structures and processes so that the organization can use what it knows to learn and to create value for its customers and community."

What is common among the definitions presented, is that $\mathrm{KM}$ is processes-driven and that it has to improve the manner in which business processes are managed [40]. According to Wiig [41], the objectives of KM are two-fold: firstly, to secure organizational viability and overall success by ensuring that the organization act as perceptively as possible and secondly, to appreciate the best value of its knowledge assets. Organizational stakeholders contribute to knowledge assets through their personal knowledge and offer organizational knowledge required for organizational improvement Therefore, it is essential to encourage their participation and contribution to the success of the organizational change [2]. Furthermore, by collecting, modelling and storing organizational knowledge, easier, smoother and more elegant organizational improvement is implemented [2]
Organizational improvement is based on critical business decisions and organizational stakeholders are making key choices based on old methods that are uninformed, with unsuccessful results [12]. This may be addressed by considering the baseline architecture that provides the current state of affairs, while looking towards a future where the business is transformed based on an outlook of what the target architecture should entail [40]. For such a transformation, an organization must stay abreast of changes in its external relationships in order to create the knowledge needed to deal with the change, to make sense of the knowledge and then to take important decisions regarding the change [31]. The change story is required to narrate the gap among the stages of knowledge creation, knowledge identification, sense-making, and decision-making. Each step of the knowing cycle provides a structured way to manage the knowledge required by an organization to successfully chart the change story [3].

In the next section we consider transformation in the context of the organization.

d) Transformation and the Organizational Context

Corporate knowledge is crucial when change occurs and is often internally focused and statically described in organizational documents at different levels of abstraction [42]. However, know-why knowledge, that is about making sense of the situation, may be gained when a change is analyzed and understood [43]. Know-why knowledge analysis entails the discovery of the reasons that lead to relevance and value add and is gained from experience and understanding of an organization articulated by business stakeholders [44]. For effective application, knowledge requires synthesizing that leads to the creation of more knowledge for decision making [31]. If a change is accepted and processed without this knowledge, it could potentially render the organization dysfunctional $[2,3]$.

When an external change forces transformation on an organization, strategic knowledge that requires sense making is required. The process of sense making ensures that people in an organization can act as a collective as they share the same understanding of the organization's goals, processes, and ways of work [31]. Sense making is therefore defined as a process that involves bracketing environmental cues, and the creation of meaning through cycles of interpretation and action that ultimately leads to enactment [45]. However, it is a subtle, sometimes informal and ongoing process, which may lead to the understatement of its value [46]. Nevertheless, it is relevant for exploring organizational knowledge and also pertinent in enhancing the understanding of the organizational environment [45-47].

The development of baseline architecture is a way in which the organization makes sense of the organization's current environment. However, the explicit efforts in the process of sense making occur when it is apparent that the future state of an organization is different to the current state [46]. Organizations that transition generate new cycles of sense-, knowledge-, and decision- making and these cycles are vital for an organization to grow and adapt [31, 48]. Organizations that manage to go through these cycles effectively are described as knowing organizations and as they exhibit a shared organizational agenda, they show a special advantage [48]. By amalgamating the cycles of sense-, knowledge-, and decision- making, a new cycle is formed known as the knowing cycle as shown in Fig. 1. When external factors trigger an organization to react and adapt, the organization must make sense of its environment, it must constantly learn and innovate, and based on what the organization's members know and believe, make decisions and act on them [3]. 


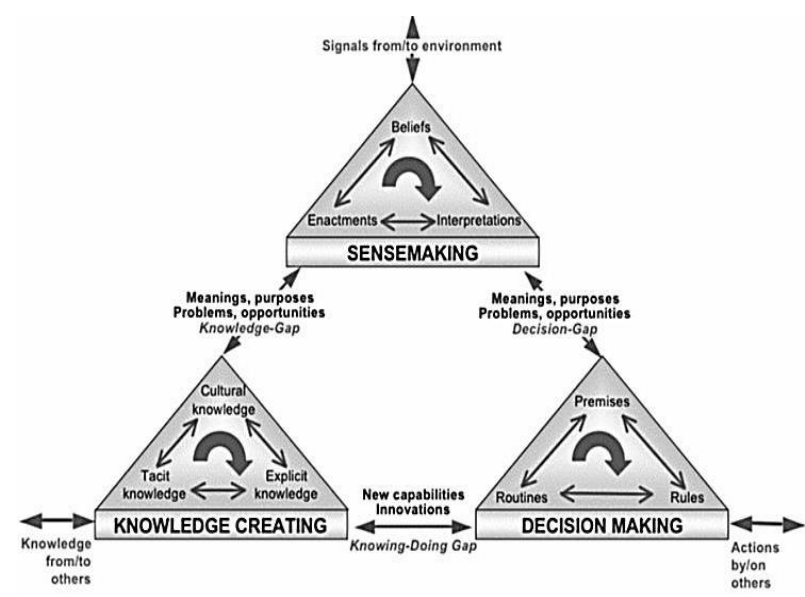

Fig. 1: The Knowing Cycle (Choo, 1998).

For sense making to take place as depicted in Fig. 1, some information and knowledge must be acquired to answer questions pertaining to what is happening in the environment that requires change, why it is happening and how it affects the organization. Processes go through performance measurement and analysis for sense making and if there is a need to improve these processes, then change is imminent [9].

In terms of decision-making, it is important to collect enough information to support the decision. Mintzberg et al. [49] define three phases for strategic decision making: (1) identification, (2) development and (3) selection. The identification phase consists of two routines: decision recognition which is about recognizing opportunities, problems, and crises; and diagnosis, where management looks to understand the effect of the external factors and determine cause and effect for the decision situation at hand. The development phase is the development and elaboration of possible solutions to a problem or crisis at hand. It consists of two routines: a search which looks for existing solutions within the organization which may be from members of the organization or in other parts of the organizational system; and secondly, the design, which is a choice between custom-made and modified solutions. The third phase, the selection phase, consists of three routines: screen, evaluation-choice, and authorization. In this phase the possible solution that has been developed in the previous phase are screened, evaluated and a final decision authorized [49].

In a fast-paced knowing organization, decision makers utilize tacit knowledge, which includes rules of thumb to make decisions that will minimize uncertainty and complexity [3]. Different situations present new opportunities for new knowledge to be interpreted and utilized creatively to solve organizational challenges [31, 49]. Either way, in terms of knowledge creation, EA is a tool that gathers explicit and tacit knowledge and social KM techniques such as knowledge cafés and communities of practice (COPs) may be used for this purpose [50]. These techniques are socially-based gatherings for people with the same interests for the purpose of sharing tacit knowledge. In turn, this type of tacit knowledge becomes strategic knowledge for the organization, which is used to develop new products and services or to improve on existing processes, thus providing competitive advantage [31,50].

In the next section we discuss the research approach followed in order to propose the business transformation framework,

\section{Business transformation investigation}

The purpose of our research was to investigate how business transformation may be achieved by using EA and the knowing cycle. In order to do so, we collected feedback via a questionnaire that included both closed and open-ended questions in order to allow the participants to share as much information as possible. In order to identify if and how knowledge can be captured effectively during the elicitation of system requirements in an HRO, a questionnaire was designed, with the following [4] sections:
- Demographic information: Gather the biographical information about each respondent, as well as the profiles of the participants.

- Concepts and meaning: Obtain meanings of concepts such as knowledge, knowledge management, and business transformation from participants.

- Enterprise architecture and business transformation: Collect feedback regarding the role of EA in enabling business transformation.

- Enterprise architecture and the knowing cycle: Gather responses on the applicability of the defined concepts in relation to EA and KM, and the effects thereof.

The responses of the multiple choice questions were tallied and analyzed. For qualitative feedback relevant parts of the data were identified and common themes were classified through a two-step process: (1) use of descriptive codes to attribute a theme to a segment of text, and (2) open coding in order to establish themes from the questionnaire data [51].

The study was conducted by targeting enterprise architects in South Africa using convenience sampling. The main aim was to engage with experts in the field of EA that were actively participating in the design or update of an enterprise architecture, both current and future states. The total number of enterprise architects targeted was 38. [22] Participants contributed to the study, yielding a response rate of $58 \%$.

The profile of the research participants is shown in Table 1. Most of the participants, $59 \%$, worked in the private sector while $41 \%$ indicated that they are based in the public or state-owned sector. The bulk of the EA projects in the private sector were within the 712 months duration range (83\%). Within the public / state-owned sector, the bulk of the EA projects were within the 13-18 months range $(47 \%)$.

Table 1: Research Participant Profile

\begin{tabular}{|c|c|c|c|c|}
\hline \multicolumn{5}{|l|}{ Profile element } \\
\hline \multirow{2}{*}{$\begin{array}{l}\text { Sector distribu- } \\
\text { tion }\end{array}$} & \multirow{4}{*}{$\begin{array}{l}\text { Public / State- } \\
\text { owned } \\
41 \% \\
\text { Public / State- } \\
\text { owned } \\
23 \%\end{array}$} & \multicolumn{3}{|l|}{ Private } \\
\hline & & $59 \%$ & & \\
\hline \multirow{2}{*}{$\begin{array}{l}\text { \# projects / sec- } \\
\text { tor }\end{array}$} & & Private & & \\
\hline & & $77 \%$ & & \\
\hline $\begin{array}{l}\text { Avg. public sec- } \\
\text { tor project }\end{array}$ & $0-6$ & $7-12$ & $13-18$ & $>18$ \\
\hline $\begin{array}{l}\text { duration in } \\
\text { months }\end{array}$ & $28 \%$ & $6 \%$ & $47 \%$ & $19 \%$ \\
\hline $\begin{array}{l}\text { Avg. private sec- } \\
\text { tor project }\end{array}$ & $0-6$ & $7-12$ & $13-18$ & $>18$ \\
\hline $\begin{array}{l}\text { duration in } \\
\text { months }\end{array}$ & $15 \%$ & $83 \%$ & $1 \%$ & $1 \%$ \\
\hline
\end{tabular}

\section{Findings and discussion}

Organizations look to change with the times and adapt to the everchanging environment in order to remain competitive as they implement transformational change [43]. The elements of business transformation, as well as the importance of KM to EA has been presented and acknowledged, as well as how to specifically manage the outputs of EA [24], [52].

In this section, we analyze and discuss the data collected regarding business transformation, EA and KM. For each section of data collected, primary themes were identified based on commonalities in the participants' responses, while emerging themes point to new ideas that the participants introduced in their responses. Lastly, we also propose a framework for business transformation using EA in association with the knowing cycle.

a) Concepts and meaning

In terms of knowledge in the context of enterprise architecture, research participants reflected primary themes such as EA artefacts, foundation architecture, enabler of enterprise architecture, business information, stakeholder insight and tacit knowledge. The emerging themes identified included enterprise nerve centre and a cross-cut- 
ting view of business. The information gathered prior to the development of an EA is important in representing the architecture of an organization. Stakeholders share their insights and their tacit knowledge of the business becomes architecture knowledge represented in artefacts. Knowledge is an enterprise nerve centre which includes the know-how of the business operations. It provides for improved decision making to deliver on change projects, thereby producing business value.

With reference to the meaning of knowledge management the primary themes identified pointed to the retention, sharing, creation, capturing, maintenance, publishing and conversion of knowledge. In addition participants highlighted the importance of the categorization of information as well as the creation of organizational value. Great emphasis was placed on keeping a well-organized, structured repository about knowledge that is easy to retrieve, maintain and that adds value to the enterprise architecture effort. Some participants indicated some emerging themes which show that effective knowledge management leads to improved company culture and insightful decision making. Another emerging theme illustrated that knowledge can be referred to as intellectual property, which requires archiving.

Common primary themes resulting from the content analysis regarding business transformation included business transformation being a process of change, in particular being a change driven by changes in the business environment. In addition research participants highlighted business transformation as a strategic journey, innovation, optimization, business modification and the alignment of people, process and technology. The emerging themes indicated concepts such as business design which refer to the changes in the structure of business. Value creation indicated that business transformation brings about value in an organization.

b) Enterprise architecture and business transformation

With the exception of only one participant, all other participants emphasized that enterprise architecture enables business transformation. All the participants highlighted the process re-engineering as a means for business transformation and that they were involved in evaluating business operations for business process improvement. Business process re-engineering is still regarded as a strategy to rapidly redesign business processes for drastic improvements.

c) Enterprise architecture and the knowing cycle

In terms of the process of sense making and the interpretation of the environment to find meaningful context, all participants supported the notion. Research participants narrated their responses referring to processes such as contextualization, understanding of business objectives, environmental understanding, assessment, scoping and the process of discovery. All participants referred to this process as the practice where an enterprise architect gets to understand the business and its objectives, as well as the scoping of the goals the organization wants to achieve. The participants justified their responses by further stating the techniques used for the sense making process. These techniques included: interviews, workshops, research, and walking the floor.

With reference to the process of knowledge creation and the collection and capturing of information which can be converted to usable knowledge, research participants pointed to the capturing of high level information, information harvesting, the modeling of the foundation architecture, knowledge development and consolidation, as well as the development of EA artefacts. The primary themes based on the responses given by the research participants show how knowledge is created in EA. Information harvesting refers to the collection of organizational information. This collected information must be captured and consolidated centrally making it explicit for ease of access and use. Develop EA artefacts refers to the development of architecture knowledge that may be reused. The emerging themes expanded on the primary themes by providing supporting detail on how the knowledge being created can be stored and managed. The concepts about which information must be stored as well as the relationships between concepts, were emphasized.

With reference to the process of decision-making and the evaluation of new knowledge to take goal-directed actions, research participants highlighted that the idea of decision making emanated from the knowledge that is created throughout the development of an EA. Primary themes in this context included customization, transformation initiatives, future simulation, knowledge-based decisions and trend interpretation. Decision making is the process in which the knowledge that has been created is used to make decisions that may change the future of the organization. Research participants highlighted several ways in which decision making in the context of EA development happens, such as trend interpretation from understanding the environmental context, and then simulating the future state of the organization. These decisions are better made when organizational knowledge is collected and known, making them knowledge-based decisions. Emerging themes on the process of decision making include new insights. Research participants emphasized the use of stakeholder engagements to develop new insights to analyze trends that affect the future direction of an organization. EA governance refers to the processes an organization puts in place to make EA decisions and enforces accountability for architecture execution.

d) Implications for the business transformation framework The evaluation of business processes for continuous improvement is part of the assessment of the current state architecture (Stoop, et al., 2016), results of which become recommendations for business transformation initiatives leading to future state. Thus, business reengineering as strategy for business transformation can be acknowledged (McKeown \& Philip, 2003). The business architecture domain is deemed a strong facilitator for changes in an organisation (Versteeg \& Bouwman, 2006) and thus informs the decisions for these changes to take place as was confirmed by the research participants. In the context of EA, the information gathered which includes process documents, policies, and procedures requires interpretation for it to be enacted. This is the process of sense making. At this stage, the architect who is interpreting the information may identify knowledge gaps that may need to be filled to enable the future state architecture. At this point, knowledge creation is required. The creation of knowledge may include the conversion of the views shared by organizational stakeholders into explicit knowledge in the form of architectural models (Lakhorst, 2009). This new knowledge that is created informs the roadmap to the future state (Lakhorst, 2009; Jonkers, et al., 2006) or forms part of the alternatives to enable decision making. When the organization acts on the decisions taken, change is enabled (Choo, 2001).

By using the themes obtained from the research participants for the application of the knowing cycle (refer Fig. 2), the knowing cycle processes and the sub-processes (refer section II.D) as defined by Choo (1998), the application of the knowing cycle can be summarized in the comparison shown in Table 2 where themes refer to the research participant inputs.

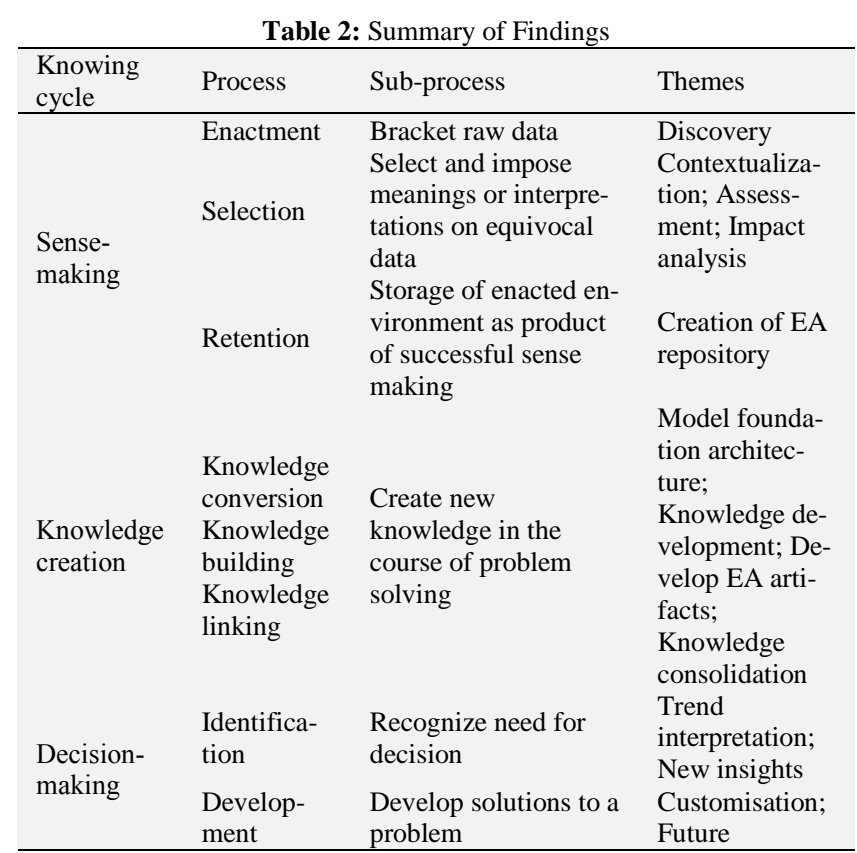




\begin{tabular}{lll}
\hline Selection & $\begin{array}{l}\text { simulation; } \\
\text { Transformation } \\
\text { initiatives } \\
\text { alternatives and } \\
\text { choose a solution }\end{array}$ & $\begin{array}{l}\text { Knowledge- } \\
\text { based decisions }\end{array}$ \\
\hline
\end{tabular}

In enactment, knowledge workers bracket raw data into equivocal data to be able to effectively interpret it. In selection, meanings are chosen to be imposed on the equivocal data. This sub-process produces an enacted environment from which an interpretation of the environment is taking place, leading to the retention of successful sense making. In EA activities derived from the themes, data is discovered from organizational stakeholders, bracketed and an analysis thereof takes place to understand the organizational environment. The retention process of the sense making mode is about the storage and retention of the meanings of the environment, thus related to the knowledge creation process and the related theme being the creation of the EA repository for storing architecture knowledge in the form of artifacts. In knowledge creation, new knowledge is created to solve organizational problems which are the identified gaps in the existing knowledge. This is essential to model the future state of an organization. Within the decision making process, new insights are identified and a need for decision making arises where the alternatives are to be evaluated. In development, the future state of the organization is simulated by developing alternative solutions through an iterative process. An evaluation of the solutions is done and an alternative solution selected based on the knowledge at hand.Based on the findings of the study reported on in previous sections, a framework for business transformation grounded in enterprise architecture and the knowing cycle is proposed in Fig. 2. For an organization adopting a change, there needs to be a blueprint that represents what is known as the as-is (baseline) architecture, the description of the manner in which the business operates currently and a starting point in identifying the enterprise components and their relationships. This model only provides enough information to facilitate the planning of what is required for improvement $(\mathrm{Ar}-$ mour, et al., 1999). The to-be (target) architecture specifies the new enterprise architecture representing "enhancements to the baseline architecture that add functions to support new operations" (Armour, et al., 1999, p. 55). The gap between the two architectures can be bridged by implementing the strategic business transformation initiatives that are identified as opportunities to improve organizational performance and ensure competitive advantage (Stoop, et al., 2016).

The processes of the different modes of the knowing cycle, namely: sense making, knowledge creation, and decision making directly use the knowledge in all of its different abstractions to identify what knowledge is required to motivate for business transformation. The role of sense making in situations of change is paramount as it helps the organization to assess what is currently going on in its environment, what the impact of the change is, and to put into context the meanings that are associated with the change. Sense making clarifies the reasons for the change to happen. The results of the sense making process leads to the creation of new organizational knowledge through innovations, introduction of new products or services, and improved business processes as opportunities for the desired future state. When a goal is in place, and possible opportunities available to choose from, management is left with the task of evaluating the possible opportunities as introduced by the knowledge creation process. At this point, management is certain that they are taking a knowledge-based decision to deliver the desire future state. In terms of sense making when developing an EA, the architects scope the EA project and establish a formal target vision of the architecture. Workshops and interviews are held to collect information about the current state of the organization such as identifying the products and services, customers, providers, business functions and processes. The stakeholder visions and expectations on the future of their enterprise can be gauged. At the end of this stage the architecture vision is in place. With reference to knowledge creation, the assessment clarifies what to document as the current state architecture, a knowledge creation step. EA artefacts in a repository are outcomes of this stage guided by reference architectures.In the context of decision making, the findings show that the architects develop future simulations, new insights and formulate transformation initiatives as part of decision making. These new and future focused formulations are part of developing a target architecture. The gaps identified between what was identified and documented as the current state architecture and the target vision produce the transformation initiatives that will enable business transformation when implemented.

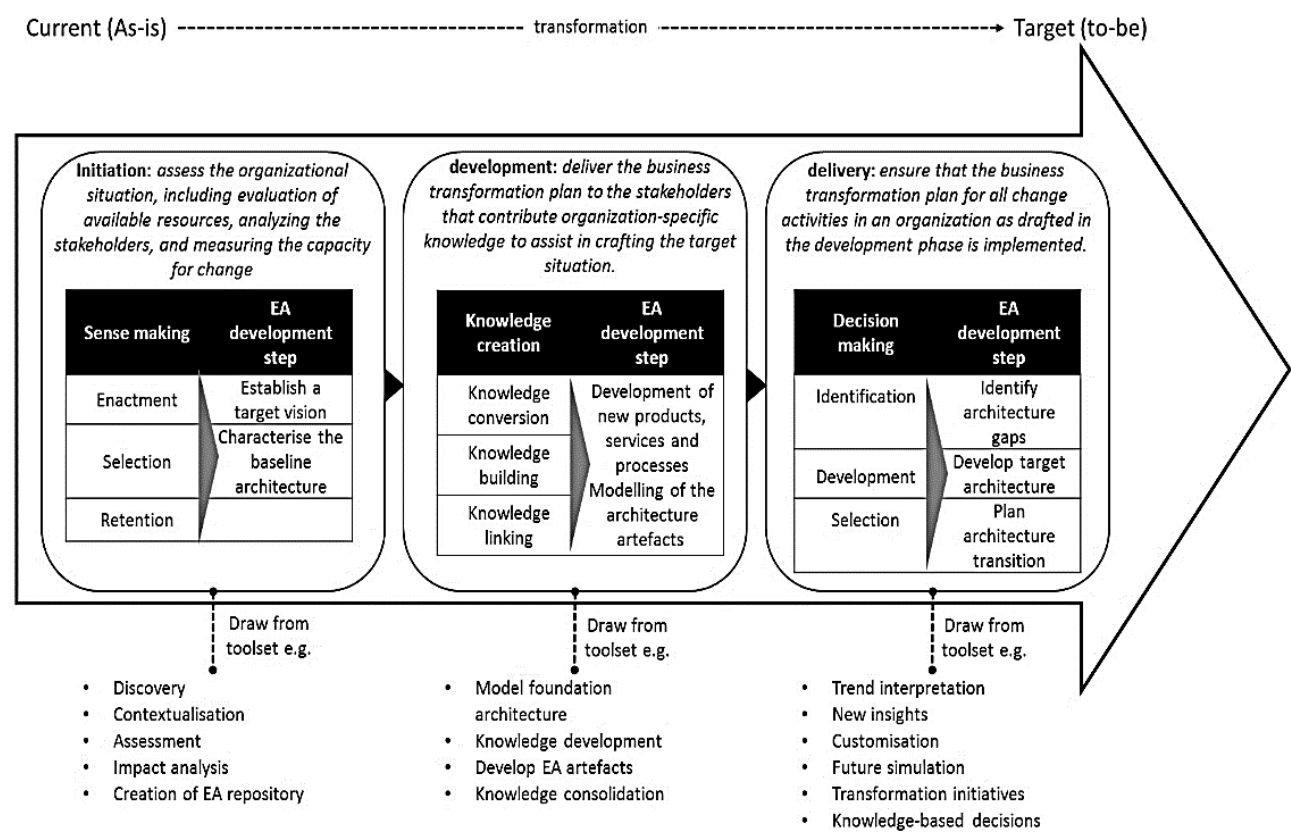

Fig. 2: Business Transformation Framework Grounded in Enterprise Architecture and the Knowing Cycle. 


\section{Conclusion}

When organizations undergo change, its goals change and these influence its mission and vision and its operating model which include products, services, distribution channels, business processes, and applications. These elements are all building blocks of an enterprise architecture, thus the organizational change translates to change in the organization's enterprise architecture blueprint. Enterprise architecture frameworks are used as knowledge collection guides, outputs of which are represented as models saved in a repository. These models address stakeholder concerns and requirements in relation to the change-taking place in the organization. In this paper, business transformation, enterprise architecture and knowledge management - in particular the knowing cycle - were explored. The experiences of enterprise architects as research participants were discovered, resulting in the proposal of a business transformation framework grounded in enterprise architecture and the knowing cycle. In addition, as a part of the framework, we suggested tools that may be used to facilitate the business transformation process. By using such a business transformation framework practicing enterprise architects may highlight the importance of the knowledge that these architects gather within the organization for enterprise architecture development, whilst also assisting them to explain to business stakeholders what value the information they share with them, has. The potential for generalization of the findings of this study is acknowledged, as the study was limited to the sample of enterprise architects from South African organizations

\section{References}

[1] Finkelstein, C., Enterprise Architecture for Integration: Rapid Delivery Methods and Technologies. Second ed. 2011, Sunnybank Hills: BookPal.

[2] Stoop, J., et al., Business Transformation Framework - To get from strategy to execution. First ed. 2016, Amersfoort: Van Haren Publishing.

[3] Choo, C.W., The knowing organisation: how organisations use information to construct meaning, create knowledge and make decisions.1998, New York: Oxford University Press.

[4] Zachman, J., Enterprise Architecture: The Issue of the Century. Database Programming and Design, 1997: p. 1-13.

[5] Wegmann, A., The systemic enterprise architecture methodology (SEAM): Business and IT alignment for competitiveness. 2002, Laboratory of Systemic Modeling: Lausanne.

[6] Veasey, P.W., Use of enterprise architecture in managing strategic change. Business Process Management, 2001. 7(5): p. 420-436.

[7] The Open Group South Africa. EA Forum.2017 [cited 20181 June] Available from: http://opengroup.co.za/ea-forum.

[8] Zachman, J.A. The Physics of Knowledge Management.1998 [cited 20181 June] Available from: www.enterprisearchitecture.dk

[9] Michela, J.L. and W.W. Burke, Organizational culture and climate in transformations for quality and innovation. , in Handbook of organizational culture and climate, N. Ashkanasy, S. Wilderom, and M. Peterson, Editors.2000, Sage: London. p. 225-244.

[10] De Long, D., Building the knowledge-based organisation: How culture drives knowledge behaviors.1997, Ernst \& Young - Centre for Business Innovation: Boston.

[11] Jonkers, H., et al., Enterprise architecture: Management tool and blueprint for the organisation. . Information Systems Frontiers, 2006 8(2): p. 63-66.

[12] Luftman, J.N., P.R. Lewis, and S.H. Oldach, Transforming the enterprise: The alignment of business and information technology strategies. IBM Systems Journal, 1993. 32(1): p. 198-221.

[13] Litvaja, I. and D. Stancekova, Decision-making, and their relation to the knowledge management - use of knowledge management in decision-making. Procedia Economics and Finance, 2015.23: p. 467472.

[14] Versteeg, G. and H. Bouwman, Business Architecture: A new paradigm to relate business strategy to ICT. . Information Systems Frontiers, 2006.Eight: p. 91-102.
[15] McKeown, I. and G. Philip, Business transformation, information technology and competitive strategies: learning to fly. International Journal of Information Management, 2003.23: p. 3-24

[16] Prahalad, C. and J. Oorsterveld, Transforming internal governance: The challenge for multinationals. Sloan Management Review, 1999. 40(3): p. 31-39

[17] Saini, A.K. and V.K. Khurana, Managing Organizational Transformation in the Era of VUCA. Journal of Management, 2015. 7(1): p 69-80.

[18] Stiles, P., A. Uhl, and P. Stratil, Meta management., in A handbook of business transformation management methodology., A. Uhl and L.A. Gollenia, Editors.2012, Gower Publishing.

[19] Ross, J.W., P. Weill, and D.C. Robertson, Enterprise Architecture as strategy.2006, Boston: Harvard Business School Press.

[20] Urbaczewski, L. and S. Mrdalj, A comparison of enterprise architecture frameworks. Issues in Information Systems. 2006. 7(2): p. 18 23.

[21] Iyamu, T., Engineering change through the domains of enterprise architecture. In Academics Conferences Limited. 2011: Toronto.

[22] Schekkerman, J., How to survive in the jungle of enterprise architecture frameworks: Creating or choosing an enterprise architecture framework. Second ed. 2004, Victoria: Trafford Publishing.

[23] Buchanan, R.D. and R.M. Soley, Aligning enterprise architecture and IT investments with corporate goals.2002 Needham: Object Management Group.

[24] Tang, A., et al., A comparative study of architecture knowledge management tools. . The Journal of Systems and Software, 2010.83: p. 352-370.

[25] Zachman, J., A framework for information systems architecture. 26(3). IBM Systems Journal, 1987. 26(3): p. 276-292.

[26] Matthee, M., P. Tobin, and P. Van der Merwe, The status quo of enterprise architecture implementation in South African financial services companies. South African Journal for Business Management, 2007. 38(1): p. 11-23.

[27] Lakhdissi, M. and B. Bounabat, toward a novel methodology for IS strategic planning.2011, Academic Conferences Limited: Toronto. p. 263

[28] Menefee, J. and D. Rudawitz. Taking Enterprise Architecture to the Next Level.2003 [cited 20181 June] Available from: www. antevorte. com/whitepapers/Taking_Enterprise_Architecture_to_the_Next_Level.pdf.

[29] Smuts, H., et al., A framework and methodology for knowledge management system implementation, in Proceedings of the 2009 Annual Research Conference of the South African Institute of Computer Scientists and Information Technologists. 2009. p. 70-79.

[30] Davenport, T.H. and L. Prusak, Working Knowledge: how organisations manage what they know. Paperback 2000 ed. 1998, Boston: Harvard Business School Press. 198.

[31] Choo, C.W., The knowing organization as learning organization. Education \& Training, 2001.43(4/5): p. 197-205.

[32] Quints, P., P. Lefrere, and G. Jones, Knowledge management: a strategic agenda. Long Range Planning, 1997. 30(3): p. 374-384.

[33] Fink, D. and G. Disterer, Knowledge Management in Professional Service Firms, in Encyclopedia of Knowledge Management, D.G. Schwartz, Editor.2011, Idea Group Inc.: London. p. 650-659.

[34] Newman, B. and K.W. Conrad, a Framework for Characterising Knowledge Management Methods, Practices and Technologies, in the Knowledge Management Theory Papers.1999, the Knowledge Management Forum: Washington.

[35] Sveiby, K.E., the New Organizational Wealth. 1997: Berrett-Koehler Publisher Inc.

[36] Meyer, M. and E. Botha, Organisation Development and Transformation in South Africa. 2000: Butterworth Publisher (Pty) Ltd.

[37] Godbout, A.J., Filtering Knowledge: Changing Information into Knowledge Assets. Journal of Systemic Knowledge Management (Journal of Knowledge Management Practice), 1999. January 1999.

[38] Lindvall, M., et al., Software Tools for Knowledge Management: A DACS State-of-the-art report. 2001 Fraunhofer Center for Experimental Software Engineering Maryland and the University of Maryland: Maryland.

[39] Choo, C.W. Knowledge Management Framework.1995 17 February 2009 [cited $2007 \quad 17$ November] Available from: http://choo.fis.utoronto.ca/.

[40] Wang, K., O.R. Hjelmervik, and B. Bremdal, Introduction to Knowledge Management:Principles and Practice.2001, Trondheim: Fagbokforlaget. 
[41] Wiig, K.M., Knowledge management: where did it come from and where will it go? Expert Systems with Applications, 1997. 13(1): p. $1-14$.

[42] Eisehardt, K. and M. Zbaracki, Strategic decision making. Strategic Management Journal, 1992.13: p. 17-37.

[43] Gilley, A., J.W. Gilley, and H.S. McMillan, Organisational change: motivation, communication, and leadership effectiveness. Performance Improvement Quarterly, 2009. 21(4): p. 75-94.

[44] Lee, Y., Why is "Know-Why" Knowledge Useful for Solving Information Quality Problems, in Americas Conference on Information Systems (AMCIS). 1996.

[45] Maitlis, S. and M.K. Christianson, Sensemaking in organisations: Taking stock and moving forward. The Academy in Management Annals, 2014. 8(1): p. 57-125.

[46] Weick, K.E., K.M. Sutcliffe, and D. Obstfeld, Organizing and the process of sensemaking. Organization Science, 2005. 16(4): p. 409421.

[47] Cecez-Kecmanovic, D., et al., Knowledge in organisations: A sensemaking view. In Organizational Knowledge, Learning, and Capabilities. 2003: Barcelona, Spain.

[48] Choo, C.W., Sensemaking, knowledge creation, and decision making: Organisational knowing as emergent strategy., in The strategic Management of Intellectual Capital and Organizational Knowledge. C.W. Choo and N. Bontis, Editors.2002, Oxford University Press: New York. p. 79-88.

[49] Mintzberg, H., D. Raisinghani, and A. Theoret, The structure of "unstructured" decision processes. Administrative Science Quartely, 1976. 21(2): p. 246-275

[50] Bou-Llusar, J.C. and M. Segarra-Cipres, Strategic knowledge transfer and its implications for competitive advantage: an integrative conceptual framework. . Journal of Knowledge Management, 2006. 10(4): p. 100-112.

[51] Vaismoradi, M. and H. Turumen, Content analysis and thematic analysis: Implication for conducting a qualitative descriptive study. Nursing and Health Sciences, 2013. 15(1): p. 398-405.

[52] Shah, H. and M.E. Kourdi, Frameworks for Enterprise Architecture IT Professional, 2007. September/October: p. 36-41. 